\title{
Colgajo libre de piel para defecto de cubrimiento de piel peneana - reporte de caso
}

\section{Skin Free Flap for Penile Skin Covering Defect - Case Report}

\author{
Dilma Alexandra Cruz Arévalo ${ }^{1} \quad$ Ana Maria Cárdenas Ortiz ${ }^{1}$ Mario Gilberto Daniel ${ }^{1}$ \\ Maria Paula Godoy Isaza ${ }^{1}$ Néstor Julián Arenas Reyes ${ }^{1}$ María Camila Bastidas Muñoz ${ }^{1}$ \\ ${ }^{1}$ Hospital Universitario Mayor-Méderi, Universidad del Rosario, \\ Bogotá, Colombia \\ Address for correspondence Dilma Alexandra Cruz Arévalo, Hospital \\ Universitario Mayor-Méderi, Universidad del Rosario, Bogotá, \\ Urol Colomb 2019;28:70-75. \\ Colombia (e-mail: alallauni@gmail.com).
}

\begin{abstract}
Resumen
Palabras Clave

- pene

- injerto de piel

- curvatura peneana
\end{abstract}

Introducción Reportar el caso de un paciente con lipogranuloma esclerosante de pene llevado a corrección de curvatura peneana compleja, con injerto de espesor parcial y posterior retracción secundaria, que generó curvatura peneana impidiendo el coito y que al momento de corrección quirúrgica, se generó un defecto de cubrimiento dérmico que requirió el uso de colgajo con piel de la pared abdominal.

Materiales y Métodos Hombre de 46 años con antecedente de resección de lipogranuloma esclerosante en cara dorsal del pene resecado en el año 2006 y disfunción eréctil en manejo con terapia intracavernosa con prostaglandina E2; con necesidad de injertos de piel de abdomen en 2 oportunidades para cubrimiento de defecto de piel, con posterior fibrosis y curvatura peneana dorsal de aproximadamente $60^{\circ}$ asociado a dolor con la erección. Llevado a corrección de curvatura peneana compleja en Hospital Universitario Mayor- Méderi. Se realiza plicatura de la túnica albugínea con técnica de Yachia hasta la verificación de corrección de curvatura dorsal en un $90 \%$. Se identifica área con defecto de cubrimiento, por lo cual se levanta colgajo en región hipogástrica abdominal y se realiza anclaje del colgajo en borde distal del defecto de cubrimiento. Seis semanas después, es llevado a segundo tiempo quirúrgico, con liberación y remodelación del colgajo y se realiza el anclaje del colgajo en el borde proximal del defecto. Tres meses después, se revalúa el paciente encontrándose cubrimiento completo del área cruenta en región dorsal del pene. Se induce erección con alprostadil intracavernoso evidenciándose curvatura peneana dorsal de menos de $5^{\circ}$.

Discusión Los pacientes con lipogranuloma esclerosante del pene generan placas en túnicas peneanas que al momento de ser resecadas, pueden requerir el uso de segmentos de piel para cubrimiento con injertos o colgajos pediculados en un terreno con alta probabilidad de daño por isquemia de dichos tejidos. En los casos en los que ocurre contracción del injerto se generan curvaturas peneanas que en caso de que impidan el coito requieren corrección de la misma. El uso de injertos autólogos o received

January 30, 2015

accepted

February 8, 2018

published online

October 5, 2018
DOI https://doi.org/

10.1055/s-0038-1642614.

ISSN 0120-789X.

e-ISSN 2027-0119.
Copyright (c) 2019, Sociedad Colombiana License terms de Urología. Publicado por Thieme Revinter Publicações Ltda., Rio de Janeiro, Brazil. Todos los derechos reservados. 


\section{Abstract}

\section{Keywords}

- penis

- skin graft

- penile curvature aloinjertos ha presentado resultados satisfactorios, sin embargo, puede requerir dos tiempos quirúrgicos para la obtención de tejido. En pacientes en quienes la piel peneana no es suficiente para cubrir el defecto, la segunda opción es la piel escrotal, que conserva características similares, así como una excelente vascularización. Cuando no se cuenta con piel escrotal en buen estado, se usa un injerto de piel de espesor total como última opción con resultados satisfactorios.

Conclusiones Actualmente, el uso de piel de abdomen en esos defectos de piel no tiene amplia acogida dada la presencia de anexos dérmicos que terminan generando un aspecto estético no óptimo, sin embargo, se evidencia en este caso, que es un tejido que se adapta fácilmente a su nueva ubicación y permite el fin último, que es lograr una adecuada función del órgano receptor.

Introduction Report the case of a patient with penile sclerosinglipogranuloma who was taken to corrrection of complex penile curvature with split-thickness graft with later secondary retraction that caused penile curvature preventing intercourse and during surgical mangement generated a skin coverge defect that required the use of free skin flap from the abdominal wall.

Material and Methods 46 year old men with a history of surgical resection of sclerosinlipogranuloma in dorsal penile resected in 2006 and erectile dysfunction managed with intracavernous therapy with prostaglandin E2; that required abdominal skin grafts in 2 opportunities for skin defect coverage, with subsequent fibrosis and dorsal penile curvature of $\sim 60$ degrees associated with pain during erection. Carried complex penile curvature correction in Hopsital Universitario Mayor - Méderi. Plication of the tunica albuginea with Yachia technique is performed until correction of dorsal curvature in $90 \%$. Coverage defect area is identified, so that flap of hypogastric abdominal region is lifted and anchored in the distal edge of coverage defect. 6 weeks later is taken to second surgical procedure, with release and remodeling of the flap and anchorage of it in the proximal edge of the defect. 3 months after, the patient is reassessed finding complete coverage of the area on the dorsal surface of the penis. Erection is induced by intracavernousalprostadil showing dorsal penile curvature of less than 5 degrees.

Discussion Patients with sclerosing penile lipogranuloma undergo plaques in penile layers that when resected may require the use of skin grafts or pedicle flaps for coverage in an area with high probability of ischemia in these tissues. In cases in which the graft contraction result in penile curvature, that in the event of preventing coitus require surgical correction thereof. The use of autologous grafts or allografts have shown satisfactory results, but may require two surgical procedures to obtain adecuate tissue. In patients in whom the penile skin is not enough to cover the defect, the second option is scrotal skin, which retains similar characteristics, as well as an excellent vascularization. When there is no adecuate scrotal skin, full thickness skin grafting as last option is used with satisfactory results.

Conclusions Currently, the use of abdominal skin in these skin defects has no wide acceptance because of the presence of dermal annexes, ending in a suboptimal aesthetic appearance, however, it is evident in this case, is a tissue that is easily adapted to its new location and allows the latter, which is to achieve adequate organ receptor function.

\section{Introducción y Objetivos}

Los pacientes con lipogranuloma esclerosante del pene generan placas en túnicas peneanas que, al momento de ser extraídas, pueden requerir resección de segmentos de piel con necesidad de cubrimiento dérmico con injertos o colgajos pediculados en un terreno con alta probabilidad de daño por isquemia de dichos tejidos. En los casos en los que se genera contracción del injerto, se generan curvaturas peneanas, y donde se impida el coito van a requerir su corrección; ese procedimiento a su vez, necesita la denudación de la piel peneana, para lograr plicar la 
túnica albugínea, lo cual lleva nuevamente a compromiso vascular y necesidad de resecar segmentos dérmicos con un consecuente defecto de cubrimiento.

A pesar de que la piel del pene tiene una amplia suplencia vascular por los vasos pudendos externos superficiales y colaterales bilaterales; el antecedente de cirugía reconstructiva de dicha piel, hace que ellos se pierdan y se requiere el uso de otros tejidos. ${ }^{1}$ Esa condición generó el inicio de uso de técnicas de reconstrucción peneana. ${ }^{2-4}$

El uso de diferentes tejidos en la reconstrucción del pene se ha descrito desde 1936 por Boragoras. ${ }^{5}$ Con respecto a defectos dérmicos se ha utilizado injertos de prepucio, ${ }^{6}$ escroto, ${ }^{7}$ abdomen inferior, colgajos pediculados de piel del muslo, de fascia lata ${ }^{8}$ y otros. Mukherjee describió el uso de injertos de piel del muslo y periné para faloplastia reconstructiva en pacientes quemados con resultados satisfactorios. ${ }^{9}$

El lipogranuloma esclerosante hace referencia a la formación de granulomas inflamatorios por cuerpo extraño en la dermis reticular y el tejido celular subcutáneo, que están rodeados de pseudoquistes de partículas lipídicas. Esa enfermedad poco común, se ha reportado como una entidad idiopática primaria o como una complicación a la inyección de sustancias oleosas sobre el tejido blando peneano; esa última también denominada parafinoma o liponecrosis. Las complicaciones surgen por la falta de degradación enzimática de los lípidos exógenos que desencadena la reacción a un cuerpo extraño. ${ }^{10}$

Los primeros reportes sobre el uso de inyecciones de minerales a base de aceites en genitales externos datan de 1899 por el cirujano Australiano Robert Gersuny, quien empleo esos agentes en la corrección de la ausencia de un testículo posterior a orquiectomía por tuberculosis genital. ${ }^{10}$ Actualmente se emplean en algunos países para el aumento del tamaño del pene y la potencia sexual, la mejoría de la función y el desempeño sexual. ${ }^{10}$

Como se mencionó anteriormente, esos pacientes desencadenan la reacción a un cuerpo extraño por los compuestos empleados, presentando un cuadro clínico que va desde la inflamación, induración y edema hasta la necrosis en los casos más severos. Las complicaciones reportadas incluyen ulceras crónicas, fistulas, deformidades secundarias a placas de fibrosis, disfunción eréctil y problemas en la micción o fimosis secundarias a la migración de los compuestos. ${ }^{1}$

El tratamiento definitivo de los parafinomas es la escisión completa de la piel y el tejido celular subcutáneo comprometido por el material foráneo y posteriormente la faloplastia. Sin embargo, aún es discutido el uso de ciclos de corticoides orales.

El uso de diferentes tejidos en la reconstrucción del pene se ha descrito desde 1936 por Boragoras. ${ }^{11}$ Los injertos de piel nativa, los colgajos libres o pediculados constituyen una buena opción para la reconstrucción peneana, con resultados favorables en cuanto a funcionalidad y erección. ${ }^{11}$ El manejo quirúrgico de las curvaturas secundarias a la formación de placas fibróticas debe ofrecerse después de haber concluido la fase inflamatoria y se debe considerar sólo en pacientes con deformidades o disfunción eréctil que impida el coito. ${ }^{11}$

El grado de deformidad, la longitud del pene y la calidad de las erecciones deben ser tenidas en cuenta en el momento de elegir la técnica quirúrgica. Las alternativas quirúrgicas para la reparación de curvaturas peneanas incluyen los procedimientos de Nesbit, Yachía y la plicatura en pacientes con buena capacidad eréctil; o la implantación de una prótesis peneana en caso de una pobre calidad de erección. ${ }^{12}$ Para reducir el acortamiento peneano postoperatorio se ha evaluado la escisión y el injerto con fascia temporalis, tunicavaginalis, dermis o material sintético, desafortunadamente esas técnicas se han asociado a un alto riesgo de disfunción eréctil postoperatoria. El uso de injertos autólogos o aloinjertos ha presentado resultados satisfactorios, sin embargo puede requerir varios tiempos quirúrgicos para la obtención de tejido. Por su lado, el material sintético puede causar inflamación postoperatoria que puede llevar a fibrosis alrededor del sitio del injerto y se asocia a una alta tasa de infección. ${ }^{12-14}$

La pérdida de piel peneana debe ser reconstruida mediante el uso del sustituto más apropiado, los mejores resultados estéticos se observan con el uso de injertos de pene o escroto, sin embargo, son los pacientes los que presentan una mayor tasa de reintervención. ${ }^{9}$ El escroto es una buena opción para obtener injertos por sus propiedades elásticas sin embargo, tiene como desventaja la diferencia en la apariencia física y la presencia de vello. En caso de no contar con piel para los injertos puede tomarse piel por colgajos libres o pediculados. ${ }^{10}$

El objetivo de este artículo es reportar el caso de un paciente con lipogranuloma esclerosante de pene que fue llevado a la corrección de curvatura peneana compleja, con injerto de espesor parcial y con posterior retracción secundaria, lo que generó curvatura peneana que impedía el coito y al momento de la corrección quirúrgica se generó un defecto de cubrimiento dérmico, por lo que requirió el uso de colgajo libre con piel de la pared abdominal.

\section{Materiales y Métodos}

Describimos el resultado de uso de colgajo de piel de pared abdominal en paciente con antecedente de lipogranuloma esclerosante del piel dorsal del pene que generaba la retracción secundaria del injerto con curvatura peneana secundaria; fue necesario ser llevado a resección de injerto fibrótico con defecto de piel secundario y corrección de curvatura peneana.

Hombre de 46 años con antecedente de inyección de material no conocido para el aumento del grosor del pene; el paciente presenta lipogranuloma esclerosante en cara dorsal del pene resecado en el año 2006 asociado a la necrosis de piel del pene y disfunción eréctil en manejo con terapia intracavernosa con prostaglandina E2, con necesidad de injertos de piel de abdomen en 2 oportunidades para cubrimiento de defecto de piel. Presenta posteriormente fibrosis y curvatura peneana dorsal de aproximadamente $60^{\circ}$ asociado a dolor con la erección e imposibilidad para el coito.

Es llevado a corrección de curvatura peneana compleja por los equipos de urología y cirugía plástica del Hospital Universitario Mayor- Méderi. Se realiza plicatura de la túnica albugínea con técnica de Yachia hasta la verificación de la 


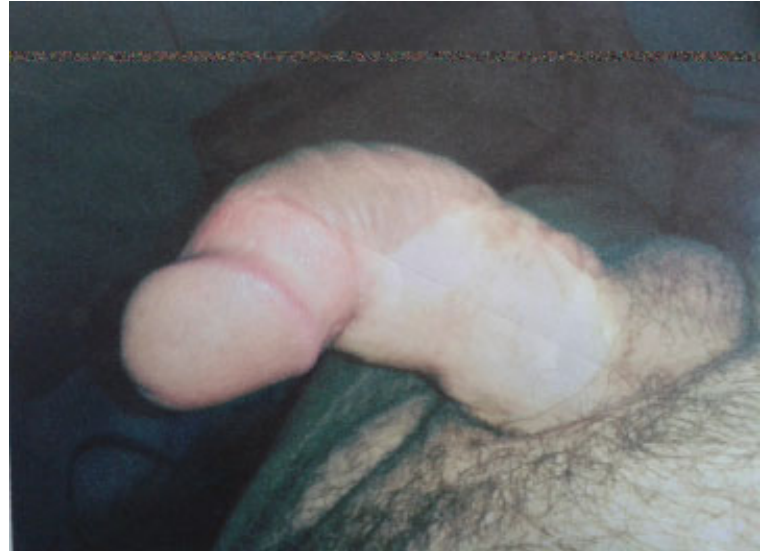

Fig. 1 Curvatura peneana prequirúrgica.

corrección de la curvatura dorsal en un $90 \%$. Se levantan colgajos de piel compuesta hacia dorso de pene y colgajos inferiores distales hasta la corona del glande. Se identifica área con defecto de cubrimiento, de aproximadamente $6 \mathrm{~cm} \times 4 \mathrm{~cm}$. Por los antecedentes de varios injertos y el riesgo de retracción del mismo, se decide realizar un colgajo de pared abdominal, esperar la inmersión del mismo y evitar el riesgo de necrosis, dado por la disminución del riego sanguíneo en el área cruenta secundario a las cirugías previas. Se levanta colgajo de piel a distancia de $8 \times 3 \mathrm{~cm}$ en la región hipogástrica abdominal y se realiza el anclaje del colgajo en borde distal del defecto de cubrimiento; se deja con sonda uretral. Seis semanas después, es llevado a segundo tiempo quirúrgico, con liberación y remodelación de colgajo de piel de pared abdominal anterior y terminando de realizar el anclaje del colgajo en el borde proximal del defecto; se cierra defecto en la piel del abdomen. No se

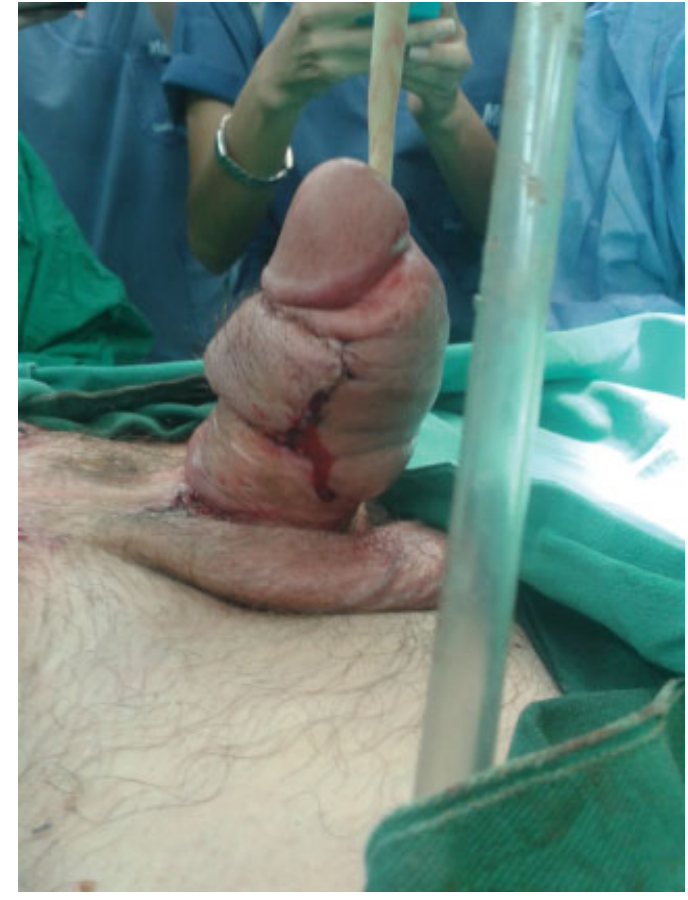

Fig. 3 Resultado del segundo tiempo quirúrgico.

realiza de otra área del cuerpo como el escroto debido a que el área del origen del colgajo iba a quedar expuesta 6 semanas; el área expuesta de la pared abdominal se cubrió con gasas vaselinadas. Tres meses después se revalúa el paciente encontrándose cubrimiento completo del área cruenta en la región dorsal del pene. Se induce erección con alprostadilin tracavernoso evidenciándose curvatura peneana dorsal de menos de $5^{\circ}$ (-Figs. 1-4).

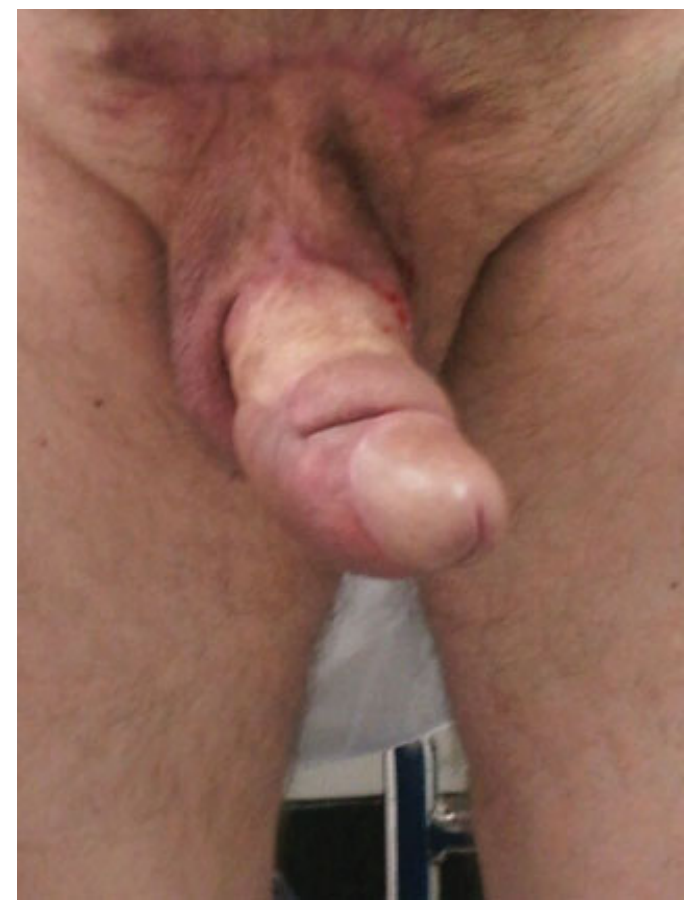

Fig. 4 Colgajo después de 3 meses del postoperatorio.

Fig. 2 Anclaje de colgajo de piel abdominal. 


\section{Discusión}

Los pacientes con lipogranuloma esclerosante del pene generan placas en túnicas peneanas que al momento de ser resecadas pueden requerir el uso de segmentos de piel para cubrimiento con injertos o colgajos pediculados en un terreno con alta probabilidad de daño por isquemia de dichos tejidos. ${ }^{11}$ En los casos en los que se genera la contracción del injerto, se generan curvaturas peneanas que en el caso de que impidan el coito, requieren su corrección, la cual necesita la denudación de piel peneana para lograr plicar la túnica albugínea, llevando nuevamente a compromiso vascular y a la necesidad de resecar segmentos dérmicos con un consecuente defecto de cubrimiento. ${ }^{11}$

La cirugía es el pilar en el manejo de las placas en túnicas peneanas $\mathrm{y}$ representa el único tratamiento efectivo en esa condición, sin embargo, no se debe realizar cirugía si la enfermedad se encuentra activa o en progresión, en donde se encuentre curvatura que no impida el coito o la presencia de una placa sin curvatura. El manejo quirúrgico se debe llevar a cabo solo después de haber concluido la fase inflamatoria y se debe considerar sólo en pacientes con deformidades o disfunción eréctil que impida el coito. ${ }^{1}$

El grado de deformidad, la longitud del pene y la calidad de las erecciones deben ser tenidas en cuenta para el momento de elegir la técnica quirúrgica. Las alternativas quirúrgicas de reconstrucción incluyen el estiramiento de la cara cóncava mediante incisión e injertos. Acortamiento de la cara cóncava, ya sea mediante los procedimientos de Nesbit o Yachía o plicatura en pacientes con buena capacidad eréctil; y la implantación de una prótesis peneana en el caso de una pobre calidad de erección. ${ }^{1}$

El acortamiento de la cara convexa se puede lograr retirando una elipse de túnica albugínea (tecnica de Nesbit), mediante la incisión horizontal de túnica albugínea (Técnica de Yachía) o mediante una plicatura peneana. ${ }^{9}$

Para reducir el acortamiento peneano postoperatorio, se ha evaluado la escisión y el injerto con fascia temporalis, tunicavaginalis, dermis o material sintético. Desafortunadamente, esas técnicas se han asociado a un alto riesgo de disfunción eréctil postoperatoria. ${ }^{1}$

Los injertos pueden ser clasificados en autólogos, aloinjertos y de material sintético. El uso de injertos autólogos o aloinjertos ha presentado resultados satisfactorios, sin embargo, puede requerir dos tiempos quirúrgicos para la obtención de tejido. Por su lado, el material sintético puede causar inflamación postoperatoria que puede llevar a fibrosis alrededor del sitio del injerto, y se asocia a una tasa alta de infeccion. ${ }^{1}$ Se han descrito técnicas de expansión de colgajo subcapsular con la instilación de solución salina hasta lograr un volumen de $600 \mathrm{cc}$, a los 4 meses se forma el colgajo y se realiza la reconstrucción del pene; en ese caso, el paciente había recibido una descarga eléctrica y tenía múltiples injertos de piel en piernas y abdomen. ${ }^{15}$

La pérdida de piel peneana debe ser reconstruida mediante el uso del sustituto más apropiado, como redistribuyendo la piel peneana restante, teniendo especial cuidado y evitando el atrapamiento peneano. En pacientes en quienes la piel peneana no es suficiente para cubrir el defecto, la segunda opción es la piel escrotal, que conserva características similares, así como una excelente vascularización. Hay reporte de 17 casos de pérdida de toda la piel del pene, donde se incluye el pene dentro del escroto y de los 2 a los 6 meses se realiza el tallaje de la piel del pene, con buenos resultados estéticos y funcionales. ${ }^{16}$ En caso de que el injerto presente vellos, esos pueden tratarse mediante depilación a láser. ${ }^{17}$

Cuando no se cuenta con piel escrotal en buen estado, se usa un injerto de piel de espesor total como opción con resultados satisfactorios, manteniendo la elasticidad y disminuyendo la probabilidad de retraerse, mejorando así la calidad de las erecciones. ${ }^{18}$ Esos colgajos donde se mantiene la vascularización, tienen excelente suplencia vascular, disminuyendo el riesgo de necrosis del colgajo. ${ }^{19,20}$

\section{Conclusión}

En el presente caso, el antecedente de uso en 2 oportunidades de injertos de espesor parcial de pared abdominal con la consecuente retracción secundaria, generaba un ambiente con mínimas probabilidades de desplazamiento del tejido ya integrado. Se consideró el injerto libre de pared abdominal la mejor elección para el cubrimiento del defecto de piel del pene posterior a corrección de curvatura peneana y resección de fibrosis de túnicas peneanas.

En ese caso, el resultado estéticamente es bueno, no se presentó retracción del colgajo y se logró corregir la curvatura. El paciente tuvo un seguimiento aproximadamente de 8 meses y se encontraba satisfecho por el resultado; no presentaba dolor con las erecciones. La respuesta a la Prostaglandina E2 estaba disminuyendo; estaba en planes de colocar prótesis peneana, sin embargo, el paciente se perdió del seguimiento.

Actualmente, el uso de piel de abdomen en esos defectos de piel no tiene amplia acogida dada la presencia de anexos dérmicos que terminan generando un aspecto estético no óptimo, sin embargo, se evidencia en este caso, que es un tejido que se adapta fácilmente a su nueva ubicación y permite el fin último que es lograr una adecuada función del órgano receptor.

\section{Responsabilidades Éticas}

Protección de personas y animales. Los autores declaran que los procedimientos seguidos se conformaron a las normas éticas del comité de experimentación humana responsable y de acuerdo con la Asociación Médica Mundial y la Declaración de Helsinki.

Confidencialidad de los datos. Los autores declaran que han seguido los protocolos de su centro de trabajo sobre la publicación de datos de pacientes.

Derecho a la privacidad y consentimiento informado. Los autores han obtenido el consentimiento informado de los pacientes y/o sujetos referidos en el artículo. Este documento obra en poder del autor de correspondencia. 


\section{Bibliografía}

1 Salgado CJ, Monstrey S, Hoebeke P, Lumen N, Dwyer M, Mardini S. Reconstruction of the penis after surgery. Urol Clin North Am 2010;37(03):379-401

2 Montealegre G. Reconstrucción total de Pene. Revista Colombiana De Urología 2011;20(03):82-88

3 Ma S, Cheng K, Liu Y, Chen F. A New Surgical Procedure for Penile Reconstruction by Combined Free Radial Forearm Flap and Dorsalis Pedis Flap. Urology 2016;97:232-237

4 Angulo JC, Arance I, Gómez-Llorens C, Esquinas C, Gómez-Martín C, Fernández-Cañamaque JL. Reconstrucción fálica total con colgajo libre antebraquial radial tras amputación peneana iatrogénica. Actas Urol Esp 2017;41(07):471-476

5 Bogoras N. Uber die volle plastiche Wiederherstellung eines zum Koitus fahigen Penis (Peni plastica totalis). Zentralbl Chir 1936; 22:1271

6 Momeni A, Abidari JM, Karanas YL. The foreskin advancement flap: an alternative technique for reconstruction of penile burns. J Plast Reconstr Aesthet Surg 2013;66(04):570-573

7 Han DS, Jang H, Youn CS, Yuk SM. A new surgical technique for concealed penis using an advanced musculocutaneous scrotal flap. BMC Urol 2015;15:54

8 Yazar Ş, Eroğlu M, Gökkaya A, Semerciöz A. The repair of complex penile defect with composite anterolateral thigh and vascularized fascia lata flap. Ulus Travma Acil Cerrahi Derg 2015;21(03):223-227

9 Mukherjee GD. On reconstruction of the penis with urethra and a dorsal skin-lined socket socket for a removable prosthesis: a new approach. Plast Reconstr Surg 1982;69(02):377-378
10 Foxton G, Vinciullo C, Tait CP, Sinniah R. Sclerosing lipogranuloma of the penis. Australas J Dermatol 2011;52(03):e12-e14

11 Ralph DJ, Garaffa G, García MA. Reconstructive surgery of the penis. Curr Opin Urol 2006;16(06):396-400

12 Perovic SV, Djinovic RP, Bumbasirevic MZ, Santucci RA, Djordjevic ML, Kourbatov D. Severe penile injuries: a problem of severity and reconstruction. BJU Int 2009;104(05):676-687

13 Moon DG, Yoo JW, Bae JH, Han CS, Kim YK, Kim JJ. Sexual function and psychological characteristics of penile paraffinoma. Asian J Androl 2003;5(03):191-194

14 Santucci RA, Zehring RD, McClure D. Petroleum jelly lipogranuloma of the penis treated with excision and native skin coverage. Urology 2000;56(02):331

15 Dong L, Dong Y, He L, et al. Penile reconstruction by preexpanded free scapular flap in severely burned patient. Ann Plast Surg 2014; 73(01, Suppl 1):S27-S30

16 Guo L, Zhang M, Zeng J, Liang P, Zhang P, Huang X. Utilities of scrotal flap for reconstruction of penile skin defects after severe burn injury. Int Urol Nephrol 2017;49(09):1593-1603

17 Summerton DJ, Campbell A, Minhas S, Ralph DJ. Reconstructive surgery in penile trauma and cancer. Nat Clin Pract Urol 2005;2 (08):391-397

18 Garaffa G, Sansalone S, Ralph DJ. Penile reconstruction. Asian J Androl 2013;15(01):16-19

19 Bryk DJ, Yamaguchi Y, Zhao LC. Tissue transfer techniques in reconstructive urology. Korean J Urol 2015;56(07):478-486

20 Glicenstein J. [The first "fillers", vaseline and paraffin. From miracle to disaster]. Ann Chir Plast Esthet 2007;52(02):157-161 